\title{
INFRARED EXCESS (IRE) AS AN INDICATOR OF PN DISTANCE
}

\author{
GEORGE JACOBY \\ Kitt Peak National Observatory, National Optical Astronomy Observatories, \\ P.O. Box 26732, Tucson, Arizona 85726, USA
}

The VLA observations presented by Pottasch et al. $(1988$, A\&A 205, 248) and Ratag et al. (1990, A\&A 233, 181) demonstrate that IRE (Pottasch et al. 1984, A\&A 138, 10) correlates very well with $F(6 \mathrm{~cm})$ for the Galactic center PN, especially when IRE is large The correlation (see figure) can be approximated by:

$$
I R E=0.0294+\frac{64.78}{F(6 \mathrm{~cm})}+\frac{321}{F(6 \mathrm{~cm})^{2}}+\frac{195.96}{F(6 \mathrm{~cm})^{3}}
$$

Since $I R E$ is a ratio of observed fluxes, it is distance independent, and we can predict $F(6 \mathrm{~cm})$, which is distance dependent, from the $I R E-F(6 \mathrm{~cm})$ relation calibrated at the GC. Comparing the predicted value for $F(6 \mathrm{~cm})$ with the observed value yields a distance to any target $\mathrm{PN}$. The relation is tightest among $\mathrm{PN}$ having high $I R E$, and is most applicable to young, dense, dusty $\mathrm{PN}$; it is not a good method to derive PN distances in general.

This method yields distances with accuracies of $15 \%$ for $60<I R E<1500,30 \%$ for $10<I R E<60$, and is unreliable for most PN which have $I R E<10$. Distances for a selection of high IRE objects agree adequately (factor of 2) with other techniques, except for the Shklovsky distances (assuming $M_{n}=0.2$, and $\epsilon=0.5$ ). Given that these objects are young, the generally large Shklovsky distances are consistent with an excessively large assumed value for the ionized mass for the stage of evolution. Inverting the Shklovsky procedure yields typical ionized masses of 0.02 to $0.10 M_{\odot}$. The dashed arrow in the figure indicates the direction and magnitude a point will move due to a factor of 2 error in the IR data.

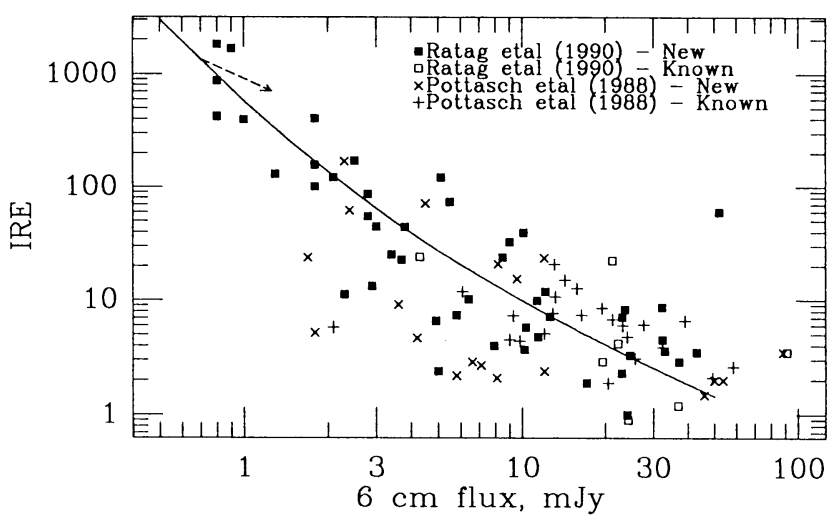

